\title{
Tagungsberichte
}

Sandra Caviola und Viola Voß

\section{Mit MALIS Zukunft gestalten - Konzepte und Strategien für die bibliothekarische Praxis}

\author{
Symposium anlässlich des 10-jährigen Bestehens des \\ berufsbegleitenden Weiterbildungsstudiengangs Bibliotheks- \\ und Informationswissenschaft (Master in Library and \\ Information Science / MALIS) an der TH Köln
}

\section{Januar - 1. Februar 2019 in Köln}

http://doi.org/10.1515/bd-2019-0036

Anlässlich des 10-jährigen Bestehens des berufsbegleitenden Weiterbildungsstudiengangs Bibliotheks- und Informationswissenschaft MALIS an der TH Köln fand am 31. Januar und 1. Februar 2019 ein Symposium mit dem Titel „Mit MALIS Zukunft gestalten - Konzepte und Strategien für die bibliothekarische Praxis“ statt, zu dem sich neben namhaften Expert^innen rund 200 Teilnehmende eingefunden hatten.

Die große thematische Bandbreite der Vorträge spiegelte die Breite der MALIS-Inhalte, die Vielfalt der Dozent*innen, von denen einige Vorträge beisteuerten, und die verschiedenen Erwartungen aus der Praxis an den Studiengang.

Nach der Begrüßung durch Studiengangsleiter Achim Oßwald sowie den Grußworten von Sylvia Heuchemer, Vizepräsidentin für Lehre und Studium der TH Köln, und Ursula Wienen, Dekanin der Fakultät für Informations- und Kommunikationswissenschaften, nahm Thomas Bürger (Honorarprofessor an der TU Dresden, bis 2018 Generaldirektor der SLUB Dresden) im ersten Vortrag die Erwartungen an Kompetenzen in Bibliotheken in Form von Rück- und Ausblicken in den Fokus. Er hinterfragte, ob das Bibliothekswesen wirklich gut auf die Digitalisierung vorbereitet ist, und sparte nicht mit kritischen Untertönen: So stelle sich z. B. die Frage, warum Bibliotheken nicht selbsterklärend gut seien, oder warum es immer noch sechs Bibliotheksverbünde in Deutschland gäbe. Google habe die Bibliotheken längst überholt, die derzeit einen „zweiten Sputnik-Schock“ erlebten, während die kommerzielle Forschung der öffentlichen Forschung „die Köpfe 
wegziehe“. Bibliotheken benötigen daher laut Bürger dringend neue Berufsbilder, die über den Data Librarian hinausgehen. Zudem sollten fachliche überinstitutionelle Kooperationen gefördert werden: „Solange selbst Doktoranden lieber googeln als in den Ressourcen der Bibliotheken zu suchen, haben wir verloren“, so eine seiner ernüchternden Thesen.

Isabella Peters (Leibniz-Informationszentrum Wirtschaft in Kiel) widmete sich dem Thema „Scholarly Communication“ bzw. der Frage, wie Bibliotheken im Kontext von Open Science auch weiterhin zur wissenschaftlichen Kommunikation beitragen können. Peters war bei der Einladung zum Vortrag über das Wörtchen „weiterhin“ gestolpert: Meine jemand vielleicht, die Bibliotheken hätten damit in letzter Zeit aufgehört, oder denken sie angesichts von Open Science nur neu darüber nach?

Peters verwies zur Einführung in Open Science auf „The Open Definition“1 und grundlegende Faktoren wie Qualität, Effizienz, Reproduzierbarkeit, Glaubwürdigkeit und Sichtbarkeit. Eine erfreuliche Entwicklung sei beispielsweise, dass es mittlerweile Stellenausschreibungen gebe, für die die Bewerber^innen darlegen müssen, wie sie bislang Open Science umgesetzt haben oder dies in Zukunft vorhaben. Weitere positive Beispiele für Open Science: Das Journal RIO² veröffentliche Projektideen, und das Projekt „Offene Doktorarbeit““3 konnte nach der Überwindung technischer und organisatorischer Schwierigkeiten erfolgreich abgeschlossen werden. Trotz solch erster Beispiele sei die praktische Umsetzung von Open Science aber immer noch schwierig. Wichtig sei es daher laut Peters, disziplinübergreifend zusammenzuarbeiten, wie dies z.B. in ihrem Kieler Team der Fall sei.

Andreas Mittrowann (Strategieberatung Nachvorndenken.de, vormals ekz) stellte unter dem Titel „Der Mensch im Mittelpunkt: Community Building und Partizipation als strategische Werkzeuge für Bibliotheken“ die Frage, was die Menschen in und von Bibliotheken brauchen: Schließlich sollte der Mensch im Mittelpunkt der bibliothekarischen Tätigkeit stehen, nicht die Medien, die lediglich ein „Werkzeug“ seien. Mittrowann fand einen humorvollen und gleichzeitig „sprechenden“ Einstieg in seinen Vortrag: Er berichtete von einem schicken Leihwagen der gehobenen Preiskategorie, der ihm einmal zur Verfügung gestellt worden war, den er aber mangels herkömmlichem Zündschloss und Schalthebel zunächst nicht ans Laufen bekam - so fühle sich technischer Wandel an! Auch für Bibliotheken stelle sich die Frage, wie man mit komplexen Situationen

1 Vgl. http://opendefinition.org. [Zugriff: 28.02.2019].

2 Vgl. https://riojournal.com. [Zugriff: 28.02.2019].

3 Vgl. https://offene-doktorarbeit.de. [Zugriff: 28.02.2019]. 
umgehen könne. Eine Alternative zur „Vereinfachung à la Trump“ sei die „Intelligenz der Vielen“, die man z.B. via Mitarbeiter-Workshops, Fokusgruppen oder Zukunftswerkstätten mit Bürgern nutzen könne. Mittrowann stellte dazu verschiedene Handlungsfelder des Community Managements in Bibliotheken vor, von der Moderation von Events bis hin zur Partizipation in Strategieprozessen.

Den Auftakt für den zweiten Tag der Veranstaltung bildete ein Vortragsblock zu ethischen Aspekten.

Eric Steinhauer (UB der Fernuniversität Hagen) widmete sich der Schattenbibliothek „SciHub“. Nach kurzer rechtlicher Einordnung des Sachverhaltes betrachtete er das Thema unter ethischen Gesichtspunkten. Dabei sei zu bedenken: Ethische Antworten könne der Einzelne nur für sich selbst geben, da Ethik von individuellen Handlungen abhänge - hier könne es also keine pauschalen oder allgemeingültigen Aussagen geben. Wenn man zudem die Perspektive von SciHub auf das gesamte wissenschaftliche Publikationswesen erweitere, müsse zum Beispiel auch gefragt werden, ob es ethisch vertretbar sei, Publikationen an Verlage zu geben, die die Autoren nicht vergüten, oder von solchen Verlagen Veröffentlichungen zu kaufen, wie es z. B. Bibliotheken tun. „Das Angebot von SciHub und dessen Nutzung markiert einen Wandel überkommener Ethiken und kann nicht an deren Maßstab gemessen werden“, so Steinhauers Fazit, der seinen Vortrag im Sinne des deutschen „Sportphilosophen“ Beckenbauer beendete: „Geht's raus und spielt's Fußball!“

Hermann Rösch (TH Köln) sprach über eines seiner Hauptforschungsthemen: die Bibliotheksethik in Deutschland. Anhand zahlreicher Beispiele führte er in ethisch relevante Themen für den Bibliotheksbereich ein: das Ausklammern bestimmter Titel aus dem Bibliotheksbestand, Videoüberwachung in Bibliotheken, Rankings in Discovery-Systemen, Log-in-Daten bei externen Datenbankanbietern, Open Access versus geistiges Eigentum, Forschungsdaten, Bibliometrie oder Fake News. Aufgaben der Bibliotheksethik als Teil der Informationsethik seien somit z.B. die Ermittlung ethischer Grundwerte, die Schaffung von Orientierung, Standardisierung und Transparenz, die Vorbildfunktion für Leitbilder bzw. Policies und die Unterstützung bei ethischen Konflikten; Instrumente der Bibliotheksethik seien z.B. die Entwicklung und Pflege von Ethikkodizes, die Einrichtung unabhängiger Ethikkommissionen, Schulungen, Fallstudiensammlungen, Beratung und nicht zuletzt die Beteiligung an der öffentlichen Diskussion.

Rösch musste nach einem „Faktencheck“ jedoch konstatieren, dass Deutschland trotz verschiedener Bemühungen nach wie vor ein „bibliotheksethisches Entwicklungsland“ darstelle: die Kodizes, die es gebe, seien weitgehend unbekannt, eine Standardisierung sei kaum zu finden, und viele Äußerungen zeugen 
von Unsicherheit der Bibliotheksbeschäftigten. Kennzeichnend für die Lage sei laut Rösch das Überwiegen der „Individualethik“ und das Fehlen einer bibliothekarischen Institutionenethik - und eine fehlende Standardisierung beim Umgang mit ethischen Fragestellungen, die dazu führe, dass immer wieder die gleichen Sachverhalte diskutiert werden.

Der folgende Block mit dem Schwerpunkt „Personalentwicklung“ wurde von Albert Bilo (UB Duisburg-Essen) eröffnet, der zum Einstieg in seinen Vortrag „Organisations- und Personalentwicklung. Anforderungen und Erwartungen an zukünftige Führungspersonen“ konstatierte, dass die Rekrutierung geeigneter Führungspersonen immer schwieriger werde. Dies habe zum einen demografische Gründe, zum anderen seien aber auch unrealistische Stellenausschreibungen und mangelnde Möglichkeiten des Erwerbs von Führungserfahrungen ursächlich. Benötigt werden daher ein neues Bild der Führungsrolle, neue Schwerpunkte beruflicher Qualifikation und verbesserte Chancen für die Mitarbeiterinnen. Dazu bedürfe es keiner Organisationsberatung, sondern vielmehr einer Veränderung von innen heraus in Richtung „postheroischer Führung“.

Bilos Empfehlungen zur Ausbildung umfassen z. B. selbstbestimmte Lehr-/ Lernformen, eine stärkere Problemorientierung, das „Training“ von Thesenbildung, Kommunikation und Moderation, lösungsorientierte Fallbeispiele oder die praktische Erfahrung von Verantwortung in „echten“ Projekten.

Dann hatten gleich drei Absolventen*innen des MALIS-Studienganges das Wort: Nicole Walger (UB Siegen), Gerald Schleiwies (Stadtbibliothek Saarbrücken) und Martina Kuth (CMS Hasche Sigle) berichteten über ihre persönliche Motivation zur Aufnahme des Studiums, den anschließenden Transfer des Erlernten in die berufliche Praxis sowie die sich aus dem Abschluss ergebenden Karriereoptionen. Nicole Walger hob hervor, dass sie im Studium viele Anregungen zu einem breiten Themenfeld bekommen habe, die sie dann in „ihre“ Bibliotheken „mitnehmen“ konnte, um Dinge voranzubringen. Gerald Schleiwies skizzierte seinen Weg „aus der Verwaltung in die Bibliothek[sverwaltung]“, während Martina Kuths Motivation für MALIS, „ihre“ Kanzleibibliothek informationswissenschaftlich voranbringen zu können, sich nach dem Studium erfüllt habe.

Das Fazit von Kuth gilt sicherlich für alle MALIS-Absolventen: „Anschließend muss man das Erlernte auch ,auf die Schiene bringen'!“

Den nächsten Themenblock eröffnete Heike Neuroth (FH Potsdam) mit dem Thema „Forschungsdaten und Langzeitarchivierung“. Die Tatsache, dass es immer noch keine einheitliche Terminologie und abgestimmte Definitionen gebe, mache das „Handling“ des Themas schwierig: Was ist z. B. „conservation“, was „preservation“, und was machen „Data Stewards“ eigentlich genau? Unter den zahlreichen Aspekten des Themenspektrums sieht Neuroth den Faktor „FAIR“ als das zukünftig beherrschende Thema. 
Für ein Raunen im Plenum sorgte ihr Hinweis auf die bemerkenswerte Ausschreibung des niedersächsischen Ministeriums für Wissenschaft und Kultur für 50 (!) unbefristete Digitalisierungsprofessuren. ${ }^{4}$

Angesichts der kaum vorhandenen bundesland-, hochschul- oder bibliotheksübergreifenden Zusammenarbeit in Sachen Forschungsdaten in Deutschland fand Neuroth deutliche Worte: Die Bibliotheken müssten sich endlich zusammensetzen und klären, was sie brauchen und wo das herkommen soll.

Achim $O \beta w a l d$ verdeutlichte den Anwesenden die Notwendigkeit von Personal Digital Archiving anhand von Szenarien aus dem Alltag wie z. B. der großen Anzahl digitaler Fotoaufnahmen via Smartphone, die viele von uns erstellen. Die Sicherung unserer digitalen Objekte sei dabei mehr als nur Datensicherung: Wir benötigen vielmehr ein umfassendes Konzept zur Erstellung, Verarbeitung, Benennung, Speicherung und Sicherung der Objekte als Teil unseres persönlichen Archivs.

Damit stelle sich die Frage, wer derzeit den Nutzer*innen die Notwendigkeit und benötigte Fertigkeiten des Personal Digital Archiving nahebringe. Computer-Clubs, Fachzeitschriften wie c't, Archive, Volkshochschulen oder das nestorNetzwerk seien hier zu nennen - aber auch Bibliotheken könnten Akteure sein. Bibliotheken seien laut $O \beta$ wald sogar in besonderem Maße dazu geeignet, weil sie nah, leicht zugänglich, vertrauenswürdig und frei von kommerziellen Interessen seien sowie über Erfahrung mit unterschiedlichen Nutzergruppen sowie über qualifiziertes Personal verfügen. Bibliotheken könnten mit der Übernahme dieser Aufgabe auch zur Sicherung des vielfältigen zivilgesellschaftlichen kulturellen Erbes beitragen.

Oßwald schloss mit einem Appell an jeden Einzelnen: „Sind wir - privat und professionell - motiviert für das Personal Digital Archiving?“

Mit einem Vortrag über Kennzahlen und Metriken als Bezugspunkte bibliothekarischer Dienstleistungen beschloss Bernhard Mittermaier (Zentralbibliothek des FZ Jülich) den Vortragsreigen. Mittermaier erläuterte anhand verschiedener Jülicher Aktivitäten anschaulich, wie das Bestandsmanagement anhand von Kennzahlen beeinflusst werde. An einem Beispiel zu potentiellen Einsparungen im Zeitschriftenbereich zeigte er einige Probleme im Spannungsfeld „Bestandsorientierung versus Nutzerorientierung“ auf. Für E-Journals gelte zum Beispiel, dass die Sinnhaftigkeit der Kennzahlen „Downloads“ und „Kosten pro Download“ neu überdacht werden müssen, da die tatsächliche Relevanz der Download-Zahlen gering sei, während die Zahlen zu den Kosten nicht alle tatsächlich anfallenden Kosten wiedergäben und die Zahlen zu den Downloads nicht

4 Siehe dazu z. B. https://idw-online.de/de/news709669. [Zugriff: 28.02.2019]. 
die gesamte Nutzung der Literatur umfassen: Schließlich sei das Publizieren in einem Journal auch eine Art der Nutzung.

Da solche herkömmlichen Kennzahlen vor allem im Bereich von OpenAccess-Zeitschriften nicht mehr ausreichen, wurde in Jülich ein „Open-AccessBarometer"5 entwickelt, das die Transformation vom Subskriptions- zum GoldOpen-Access Modell unterstützen soll. Mittermeier sieht das FZ Jülich hier auf einem guten Weg, aber noch nicht am Ziel.

Zum Abschluss der gelungenen Veranstaltung konnte das Kollegium des MALIS-Studiengangs Achim Oßwald und Herrmann Rösch anlässlich ihrer Verabschiedung in den (Un-)Ruhestand jeweils mit einer eigenen Festschrift überraschen. Die beiden Bände, die in Kürze auch online frei verfügbar sein werden, spiegeln die Arbeitsschwerpunkte der beiden Wissenschaftler und Dozenten wider: „Bibliotheksentwicklung im Netzwerk von Menschen, Informationstechnologie und Nachhaltigkeit“ (Oßwald) und „Die Bibliothek im Spannungsfeld: Geschichte - Dienstleistungen - Werte“ (Rösch).

Das Symposium bot neben den abwechslungsreichen und humorvollen Vorträgen auch viel Gelegenheit zum Austausch unter den ehemaligen MALISTeilnehmer^innen, den Dozenten ${ }^{\star}$ innen und den weiteren Teilnehmer*innen.

Zur Nachlese der Veranstaltung sei auf die Vortragsfolien ${ }^{6}$ und die zahlreichen Tweets unter den Hashtags \#10jahremalis ${ }^{7}$ und \#malissymposium ${ }^{8}$ verwiesen.

5 Vgl. http://www.fz-juelich.de/zb/DE/Leistungen/Open_Access/oa_barometer/oa_barometer_ node.html. [Zugriff: 28.02.2019].

6 Vgl. https://www.th-koeln.de/studium/bibliotheks--und-informationswissenschaft-master-inlibrary-and-information-science---symposium-2019_58694.php. [Zugriff: 28.02.2019].

7 Vgl. https://twitter.com/hashtag/10jahremalis?f=tweets\&vertical=default\&src=hash. [Zugriff: 28.02.2019].

8 Vgl. https://twitter.com/search?f=tweets\&vertical=default\&q=\%23malissymposium\&src=typd. [Zugriff: 28.02.2019]. 